\title{
Phase Field Simulation of the Effect of Anisotropy in Grain Boundary Energy on Growth kinetics and Morphology of Grain Structure
}

\author{
Yoshihiro Suwa $^{1}$ and Yoshiyuki Saito ${ }^{2}$ \\ ${ }^{1}$ Research Center for Advanced Science and Technology, The University of Tokyo, Tokyo 153-8904, Japan \\ ${ }^{2}$ Department of Materials Science and Engineering, Waseda University, Tokyo 169-8555, Japan
}

Effect of anisotropy in grain boundary energy on kinetics of grain growth and topological properties of grain structure in two dimensions was simulated by the phase field model. Misorientation distribution in a system with anisotropic grain boundary energy is found to be timedependent. Fraction of low angle grains boundaries increases with time and high angle grains disappear fast. The average area is found to be proportional to time in both isotropic and anisotropic cases. The anisotropy in grain boundary energy delays the growth rate. The scaled grain size and the edge number distributions become time-independent in both isotropic and anisotropic cases. Anisotropy in grain boundary energy broadens the scaled grain size and the edge number distributions. The characteristics of the size distribution can be represented by the variation in a parameter, called microstructural entropy. The nearest neighbor face correlations obtained by the simulated grain structures with isotropic and anisotropic grain boundary energies are quite similar to the Aboav-Weaire relation.

(Received December 21, 2004; Accepted March 31, 2005; Published June 15, 2005)

Keywords: phase field model, grain growth, anisotropy, size distribution, edge number distribution

\section{Introduction}

Modeling of kinetics of grain growth is essentially important for materials design of structural materials. Due to the difficulty of incorporating topological features into analytical theories of grain growth directly ${ }^{1-5)}$ there has been increasing interest in the use of computer simulations to study behavior of grain growth. A variety of models have been proposed during the past two decades. ${ }^{6-14)}$ Among these models, the Monte Carlo and the phase field models ${ }^{11-14)}$ are the most robust and versatile and certainly the most highly developed and widely applied. The feature of the Monte Carlo model is that grain boundaries are described as sharp interfaces with zero thickness. This makes modeling of grain growth simpler, however it has a disadvantage in predicting microstructural evolutions in practical materials.

The phase field model has been successfully applied to simulation of temporal and spatial evolution of microstructure. This model represents temporal evolutions in the chemical composition, crystallographic, and structural fields. In the phase field model phase boundaries are assumed to be diffused with finite thickness. The free energy density functional is defined to have continuous local order parameters. Temporal evolution of the whole microstructure is calculated by the temporal and spatial changes of local order parameters. The polycrystalline microstructure is described by a set of orientation field variables; $\eta_{1}(r), \eta_{2}(r), \ldots, \eta_{p}(r)$. The interfacial energy of a material is related to parameters in fundamental equations of the phase field models, i.e. the gradient energy coefficient, $\kappa$, and a parameter in a local free energy function, $a_{3}$.

When the motion of grain boundary is driven by its curvature, it is known that the migration speed of a small segment of the boundary, $v$, can be written as:

$$
v=L \gamma\left(\lambda_{1}+\lambda_{2}\right),
$$

where $L$ is the Onsager's phenomenological coefficient, $\lambda_{1}+$ $\lambda_{2}$ is the local mean curvature and $\gamma$ is the grain boundary energy density. Although grain growth has been analyzed mostly in the isotropic limit, where both $L$ and $\gamma$ are taken to be constant, it is well known that both the energy and mobility of grain boundaries are strongly anisotropic.

In this paper, the effect of anisotropy in the grain boundary energy on growth kinetics and topological properties of grain structure has been investigated. The effect of anisotropy in grain boundary energy on grain growth has been investigated by the Monte Carlo method. It is known that anisotropy in grain boundary energy broaden the grain size and face number distributions. We will investigate kinetics of grain growth in two dimensional lattice with anisotropic grain boundary energy by the phase field model.

\section{Simulation Model}

In the phase field model for grain growth of polycrystalline materials, microstructure of polycrystalline materials is described by set of orientation field variables, $\eta_{1}(r)$, $\eta_{2}(r), \ldots, \eta_{p}(r)$, where $\eta_{i}(r) \quad(i=1,2, \ldots, p)$ are called orientation field variables that distinguish different orientations of grains and $p$ is the integer number of possible orientations. Within the grain labeled by $\eta_{1}$, the absolute value for $\eta_{1}$ is 1 while all other $\eta_{i}$ for $i \neq 1$ is zero. Across the grain boundaries between the grain $\eta_{1}$, and its neighbor grains, the absolute value of $\eta_{1}$ changes continuously from 1 to 0 . According to Cahn and Hilliard, ${ }^{15)}$ the total free energy functional of an inhomogeneous system is given by

$$
F=\int\left[f_{0}\left(\eta_{1}(r), \eta_{2}(r), \ldots, \eta_{p}(r)\right)+\sum_{i=1}^{p} \frac{\kappa}{2}\left(\nabla \eta_{i}(r)\right)^{2}\right] d^{3} r
$$

where $f_{0}$ is the local free energy density which is a function of orientation field variables, $\eta_{i}(r)$, and $\kappa$ is the gradient energy coefficient. The spatial and temporal evolutions of orientation field variables are described by the time-dependent Ginzburg-Landau equations for nonconserved order parameter: ${ }^{16)}$ 


$$
\frac{\partial \eta_{i}(r, t)}{\partial t}=-L_{i} \frac{\delta F}{\delta \eta_{i}(r, t)}, \quad(i=1,2, \ldots, p)
$$

where $L_{i}$ are the Onsager's phenomenological coefficients. We used the Ginzburg-Landau type free energy density functional for the present simulation,

$$
\begin{aligned}
f_{0}\left(\eta_{1}(r), \eta_{2}(r), \ldots, \eta_{p}(r)\right)= & \sum_{i=1}^{p}\left(-\frac{a_{1}}{2} \eta_{i}^{2}+\frac{a_{2}}{4} \eta_{i}^{4}\right) \\
& +a_{3} \sum_{i=1}^{p} \sum_{j \neq i}^{p} \eta_{i}^{2} \eta_{j}^{2},
\end{aligned}
$$

where $a_{1}, a_{2}$ and $a_{3}$ are phenomenological parameters. The only requirement for $f_{0}$ is that it has $2 p$ minima with equal well depth at $\left(\eta_{1}, \eta_{2}, \ldots, \eta_{p}\right)=(1,0, \ldots, 0),(0,1, \ldots, 0), \ldots$, $(0,0, \ldots, 1),(-1,0, \ldots, 0),(0,-1, \ldots, 0), \ldots,(0,0, \ldots,-1)$. Therefore, $a_{3}$ has to be greater than $a_{2} / 2$ when we assume $a_{1}=1, a_{2}=1$. In order to simulate the ideal case of uniform mobilities and energies, we set each order parameter equal to its absolute value, effectively restricting the available order parameter space to that containing only the $p$ degenerate minima of $f_{0} \cdot{ }^{13)}$

When $L, \kappa$ and $a_{3}$ are assumed to be constant, the above equations describe isotropic grain growth. Anisotropy is incorporated by making $L, \kappa$ and $a_{3}$ misorientation under constraint of constant grain boundary width ${ }^{17)}$ as follows: According to the analysis of Cahn and Hilliard, ${ }^{15)}$ there exists a simple relation between the gradient energy coefficient $\kappa$ and the grain boundary energy $E_{\mathrm{gb}}$ as $\kappa \propto E_{\mathrm{gb}}^{2}$. So simply taking $\kappa \propto E_{\mathrm{gb}}^{2}$ will alter the grain boundary thickness and, may affect the simulation results. It is well known that the thickness of grain boundary $l$ in the phase field approach is ${ }^{18)}$

$$
l \approx \sqrt{\kappa /(\Delta f)_{\max }}
$$

where $(\Delta f)_{\max }$ is the maximum height of the barrier in the free energy density between two minima and grain boundary energy is given by ${ }^{18)}$

$$
E_{\mathrm{gb}} \approx \sqrt{\kappa \cdot(\Delta f)_{\max }}
$$

In the simulations by changing both $\kappa$ and $(\Delta f)_{\max }$ and simultaneously and keeping the ratio $\kappa /(\Delta f)_{\max }$ constant, one can simulate boundaries of the same thickness but different energies. The relative height of the barrier height corresponding to the energy $E_{\mathrm{gb}}$ is given by: ${ }^{17)}$

$$
(\Delta f)_{\max }=\frac{E_{\mathrm{gb}}}{E_{\mathrm{gb}}^{(0)}}(\Delta f)_{\max }^{(0)}
$$

where $(\Delta f)_{\max }^{(0)}$ is a certain choice of value for barrier heights that gives desired boundary thickness $l^{(0)}$ and $E_{\mathrm{gb}}^{(0)}$ is a correspond value for the grain boundary energy. In this case gradient coefficient is chosen to be a value $\kappa^{(0)}$. Changes in the height of the barrier can be accompanied by varying the coefficient $a_{3}$.

According to Ma et al. ${ }^{19)}$ we characterize the misorietation field corresponding to an arbitrary distribution for grain boundaries in system

$$
\theta(\vec{r})=\frac{\sum_{i, j \neq i}^{p} \eta_{i}^{2} \eta_{j}^{2} \theta_{i j}}{\sum_{i, j \neq i}^{p} \eta_{i}^{2} \eta_{j}^{2}}
$$

where $\theta_{i j}$ is the misorientation angle between grain $i$ and grain $j$ with orientations $\eta_{i}$ and $\eta_{j}$ eq. (8) assign a constant misorientation angle within the grain boundary region between grain $i$ and grain $j$ with value of the angle $\theta_{i j}$, given by a predetermined look-up table, and yield a weighed-mean misorientation at junction. To determine the detailed form of $L$ and $\kappa$, it is necessary to know dependences of grain boundary energy and mobility on misorientatation.

In a simple dislocation model the energy of a low angle tilt boundary between two cubic crystals has been obtained as a function of misorientation and inclination. ${ }^{20)}$

$$
\gamma(\theta)=\gamma_{0} \frac{\theta}{\theta_{\mathrm{m}}}\left(1-\ln \left(\frac{\theta}{\theta_{\mathrm{m}}}\right)\right)
$$

where $\theta_{\mathrm{m}}$ is the maximum angle at which Read-Shockley formula holds and $\gamma_{0}$ is constant. The value of $\theta_{\mathrm{m}}$ is assumed to be $18^{\circ}$ in the simulation.

In this simulation the mobility anisotropy is not taken into consideration,

$$
L=L_{0}
$$

It is reported that the mobility anisotropy does not significantly change grain growth kinetics and topological properties of simulated grain structure. ${ }^{17)}$

For the purpose of simulating the grain growth kinetics, the set of kinetic eq. (3) have to be solved numerically by discretizing them in space and time. In order to minimize the lattice anisotropy, the Laplacian is discretized by the following equation,

$$
\nabla^{2} \eta_{i}=\frac{1}{(\Delta x)^{2}}\left[\frac{1}{2} \sum_{j}\left(\eta_{j}-\eta_{i}\right)+\frac{1}{4} \sum_{k}\left(\eta_{k}-\eta_{i}\right)\right],
$$

where $\Delta x$ is the discretizing grid size, $j$ represents the first nearest neighbors of site $i$ and $k$ represents the second nearest neighbors of site $i$. For discretization with respect to time, we used the simple explicit Euler equation,

$$
\eta_{i}(t+\Delta t)=\eta_{i}(t)+\frac{d \eta_{i}}{d t} \times \Delta t,
$$

where $\Delta t$ is the time step for integration.

\section{Result and Discussion}

\subsection{Microstrucural evolution and kinetics of grain growth}

Simulation were performed on 2-dimensional lattice with size of $N=2100^{2}$ and the number of orientations of $p=72$. The lattice step size $\Delta x$ was set to be 1.0 and a time step $\Delta t$ of 0.05 was employed. All simulations were performed on the lattice systems with periodic boundary condition. In order to prevent large discontinuous change in grain size by coarsening of grains having the same orientation, the nucleation sites were situated so that grains with same orientation are located at distance of a pre-set minimum distance in each phase field. To visualize the microstructure evolution using the orientation field variables, the following function was defined:

$$
\varphi(r)=\sum_{i=1}^{p} \eta_{i}^{2}(r)
$$




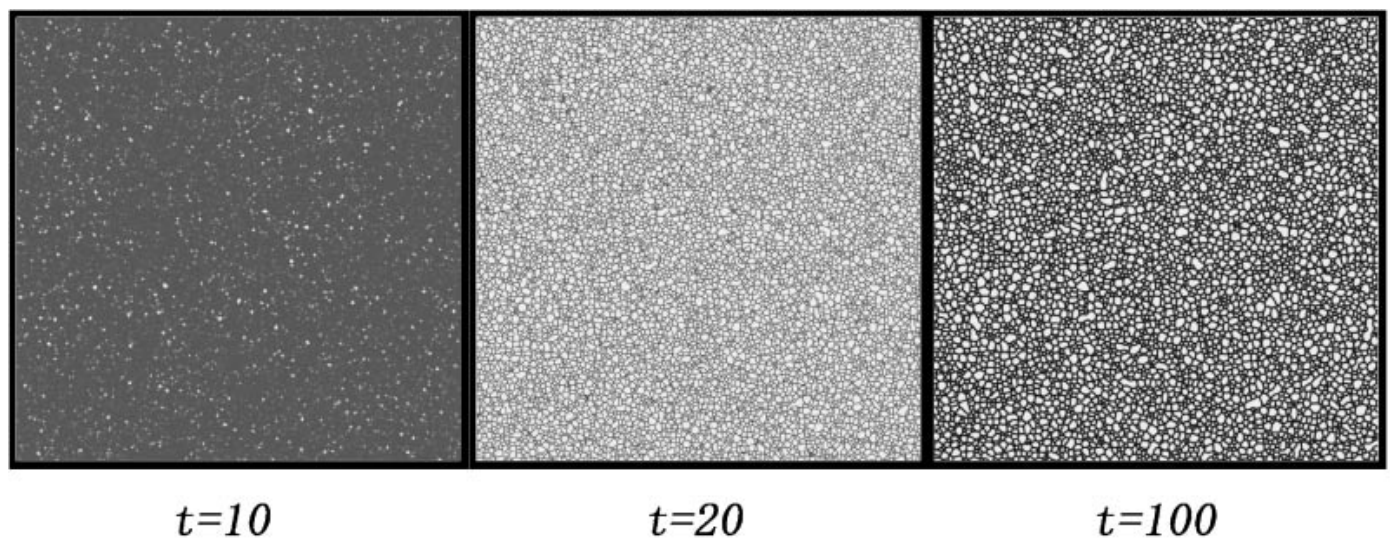

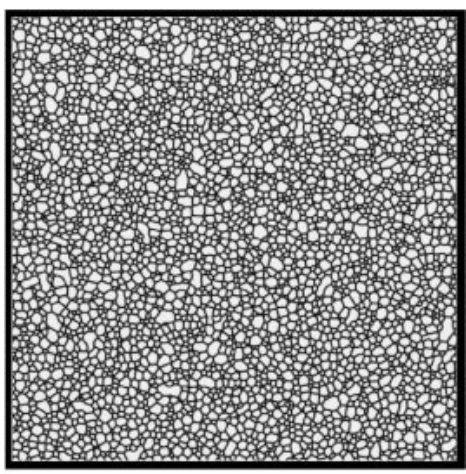

$t=200$

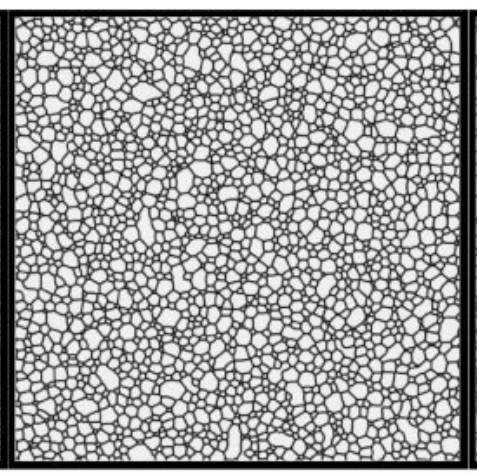

$t=500$

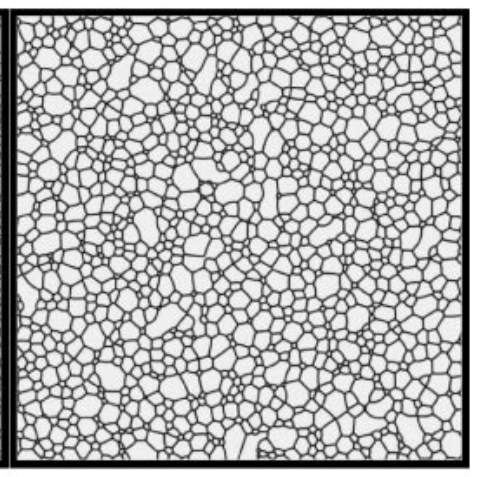

$t=1000$

Fig. 1 Simulated microstructural evolution in $2100 \times 2100$ cells in isotropic case.

which takes on a value of unity within individual grains and smaller values in the core regeions of the boundaries. ${ }^{13,14)}$ If we map the value of $\varphi$ to a specturm of graylevels, then we obtain images like that of Fig. 1, in which the grain boundaries appears as dark regions separating individual grains. The topological properties such as number of side, cross-sectional length, or area can be evaluated directly by choosing a threshold value in $\varphi$ to establish the boundary positions. In this manner, it is possible to quantify the evolution of local and averaged topological grain properties during coarsening.

Figure 1 shows temporal evolution of microstructure obtained from simulation for isotropic grain boundary. Typical microstructure during grain growth in systems with anisotropic grain boundary energy is shown in Fig. 2. There is an increase in number of grain boundaries which is considered to have low grain boundary energy. It is clear that in the anisotropic case grain shape anisotropy develops. Misorientation distribution in systems with isotropic grain boundary and anisotropic grain boundary is shown in Fig. 3 . Simulations were started with a uniform misorientation distribution, which provide an equal probability to find grain boundaries of any misorientation. The distribution in the isotropic case is time-independent because all boundary properties are not time-dependent. When grain boundary energy is anisotropic, fraction of low angle (low energy boundary) boundaries increases significantly during grain growth. This suggests that high angle (high energy) boundaries disappear at a faster rate.

The average grain area versus time for isotropic and anisotropic cases is shown in Fig. 4. in both cases, the average area is found to be proportional to time. Growth rate for anisotropic case is slower than that in isotropic case. This is considered to be due to the fact that a number of lower energy boundaries in anisotropic case increases with time. The migration speed of a small segment of the boundary is known to be proportional to grain boundary energy. ${ }^{1)}$

\subsection{Grain size and edge number distributions}

In order to get the grain size and edge number distributions 3 runs of simulation were performed. After a short transient time, the grain size distribution becomes time-independent. The scaled grain size distribution for isotropic and anisotropic cases is shown in Fig. 5. The distribution for anisotropic case is slightly broader than that for isotropic case, i.e. when grain boundary is anisotropic, fractions of small and large grains become larger.

To evaluate profile of the grain size distribution, a parameter $S$ is defined as

$$
S=\frac{\Sigma_{i} g_{i} \ln \left(g_{i}\right)}{\Sigma_{i} g_{i}},
$$

where $g_{i}$ is the value of normalized grain size defined by $g_{i}=R_{i} / R_{\mathrm{av}}$, in the $i$-th size group of $R_{i} / R_{\mathrm{av}}$. From the analogy to the entropy of ideal mixing, $S$ is called microstructural entropy. ${ }^{21)}$ When the size distribution has a narrow peak, the value of $S$ is expected to be small. When the distribution becomes flat or widely scattered, $S$ pass through an extreme. As shown in Fig. 6, the value of $S$ for anisotropic case is larger. The characteristics of size distribution profile 


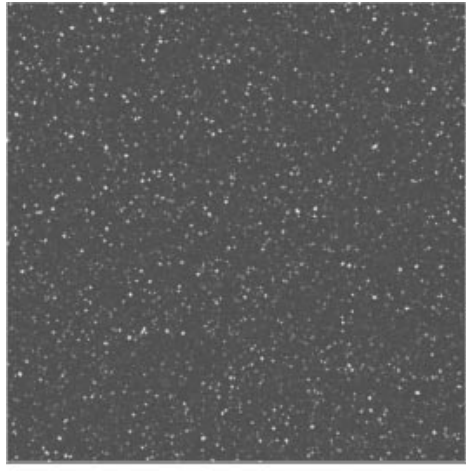

$$
t=10
$$

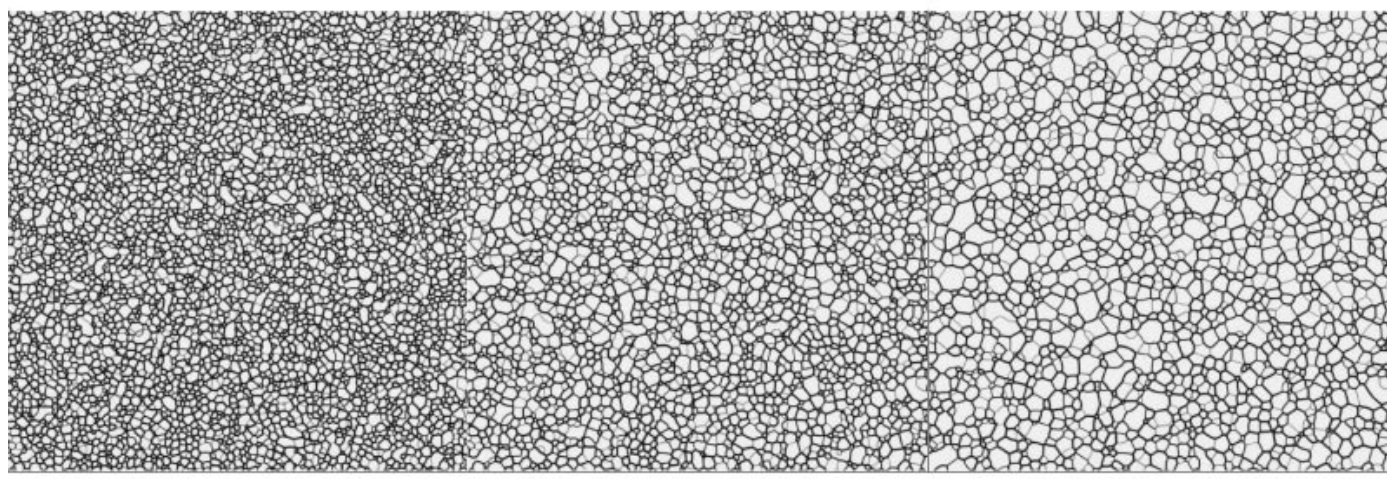

$t=200$

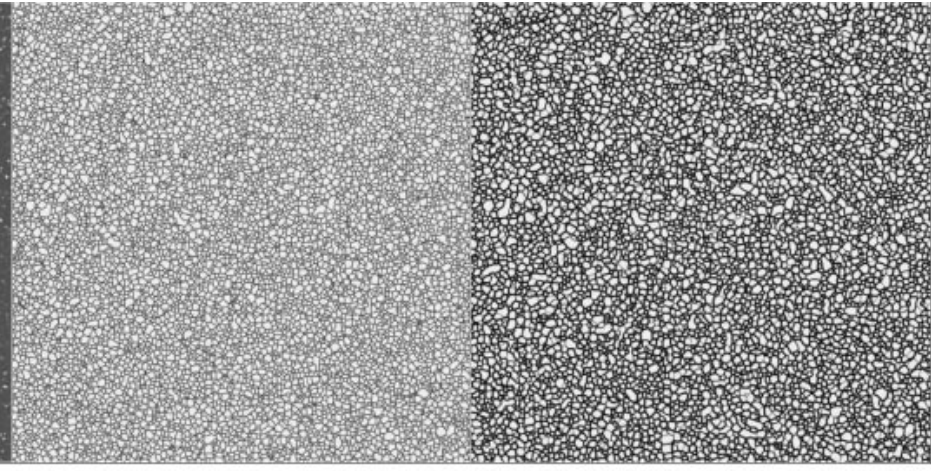

$t=20$

$$
t=100
$$

Fig. 2 Simulated microstructural evolution in $2100 \times 2100$ cells in anisotropic case.

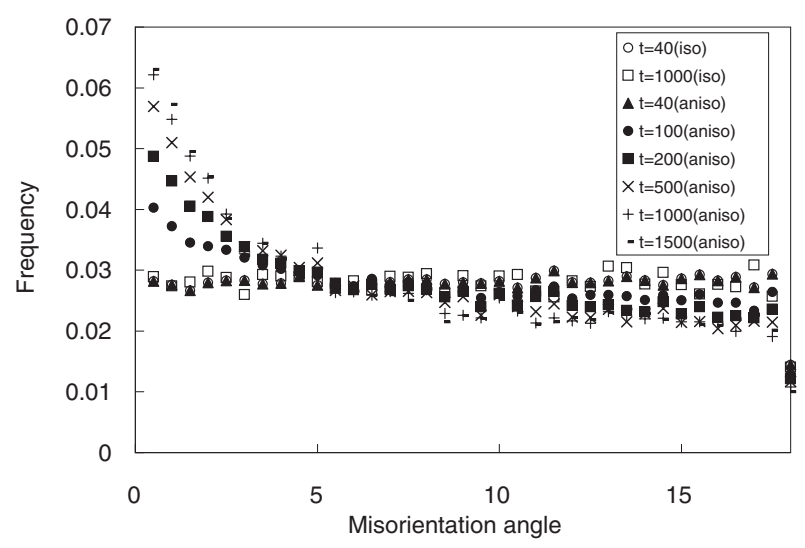

Fig. 3 Variations in misorientation distribution in isotropic and anisotropic cases during grain growth.

may be represented by the value $S$ efficiently.

The distribtuions of the edge number $N_{\mathrm{e}}$, for individual grains of the simulated microstructures is calculated. The distribution becomes time-invariant at the longer time. The frequency increase rapidly for a small number of edges and peaks at a vlaue of 5 or 6 and decays quickly. Figure 7 shows comparison of the edge number distribution for the system with anisotropic grain boundary energy with that for the isotropic system. As shown in Fig. 7 the edge number distribution for grain structure with anisotropic grain boundary is slightly broader than that for the isotropic case.

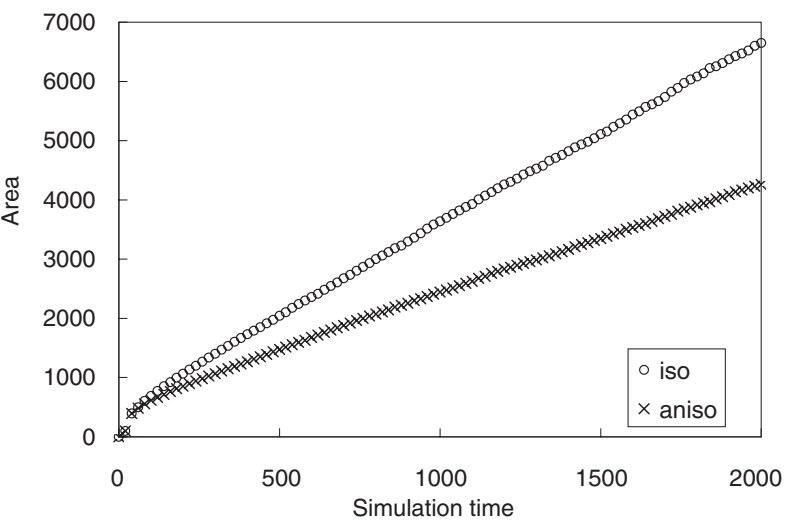

Fig. 4 Average grain area versus time in isotropic and anisotropic cases.

\subsection{Nearest neighbor edges correlation}

The relationship between the average number edges of grain adjacent to an $N$-edged grain, $m\left(N_{\mathrm{e}}\right)$, and the edge number of grains, $N_{\mathrm{e}}$, is shown in Fig. 8. The linear relation equation known as the Aboav-Weaire relations ${ }^{22,23)}$ is obtained between $m\left(N_{\mathrm{e}}\right)$ and $N_{\mathrm{e}}$.

$$
\begin{array}{rlr}
m\left(N_{\mathrm{e}}\right) & =5.038+\frac{8.427}{N_{\mathrm{e}}} & \text { isotropic case } \\
& =5.023+\frac{8.223}{N_{\mathrm{e}}} \quad \text { anisotropic case }
\end{array}
$$

Empirical equation given by $\mathrm{Aboav}^{22)}$ is 


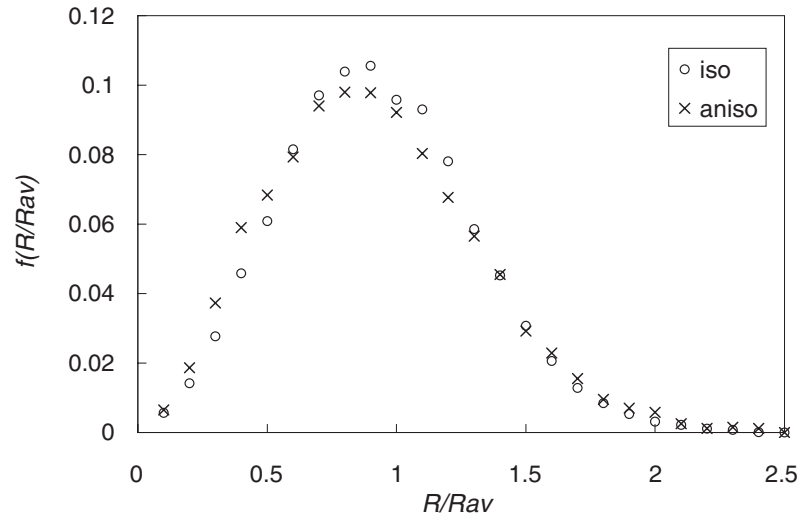

Fig. 5 Comparison of the scaled grain size distribution function in anisotropic case with that in isotropic case.

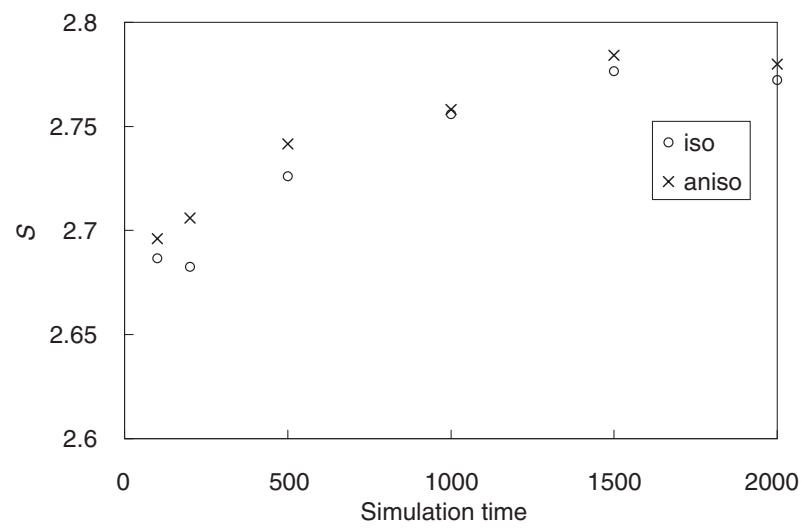

Fig. 6 Variation in the microstructural entropy, $S$, with time in isotropic and anisotropic cases.

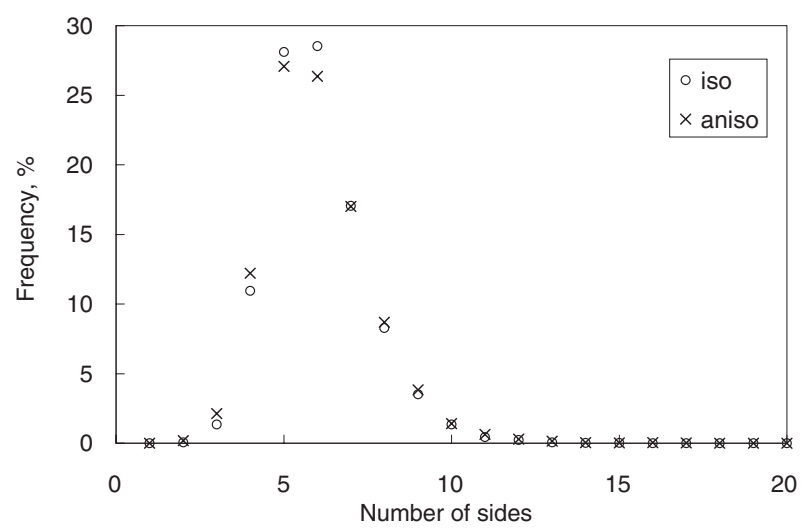

Fig. 7 Comparison of the edge number distribution function in anisotropic case with that in isotropic case.

$$
m\left(N_{\mathrm{e}}\right)=5+\frac{8}{N_{\mathrm{e}}}
$$

This can be generalized to a relation ${ }^{24)}$

$$
m\left(N_{\mathrm{e}}\right)=5+\frac{6+\mu}{N_{\mathrm{e}}},
$$

where $\mu$ is the second moment of the side of grains. Differences between the parameters in the Aboav-Weaire

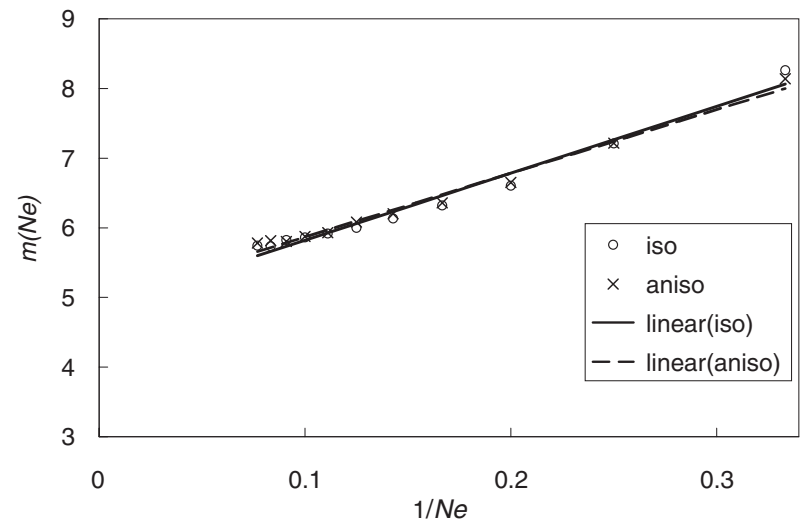

Fig. 8 Comparison of the nearest neighbor face number correlation in anisotropic case with that in isotropic case.

type empirical equation in the isotropic case and those in the anisotropic case are not significant. As is pointed out by Holm et al. ${ }^{25)}$ lattice anisotropy affects the second moment $\mu$. In this simulation the lattice anisotropy is so small that our empirical equation is quite similar to Aboav's relation.

\subsection{Comparison of various simulation results}

The Monte Carlo simulation results in two dimensions ${ }^{26)}$ indicate that the grain size and grain edge distributions become broad in systems with anisotropic grain boundary energy. In these model the broadening of the distributions is attributed to disappearance of high energy grains by grain boundary wetting. Similar result is obtained by a 3-demensional Monte Carlo simulation. ${ }^{27)}$ This result is in good agreement with present simulation results.

Kazaryan et al. ${ }^{17)}$ investigated effect of anisotropy and mobility on grain growth. Their result indicates that grain boundary energy anisotropy significantly delays grain growth and broadens edge number distribution. They also showed that distribution of misorientation in anisotropic case is timedependent. The above results are in good agreement with those obtained by this simulation. However, they indicate that anisotropy in grain boundary energy does not affect the grain size distribution, which does not coincide with the present simulation. Further investigation is necessary.

\section{Summary}

Effect of anisotropy in grain boundary energy on temporal evolution and morphology of grain structure in two dimensions was simulated by the phase field model.

The following results are obtained.

(1) Misorientation distribution in a system with anisotropic grain boundary energy is found to be time-dependent. Fraction of low angle grains boundaries increases with time and high angle grains disappear fast. The average area is found to be proportional to time in isotropic and anisotropic cases. The anisotropy in grain boundary energy delays growth rate. The scaled grain size and the edge number distributions become time-independent in both isotropic and anisotropic cases.

(2) Anisotropy in grain boundary energy broadens the scaled grain size and the edge number distributions. The 
characteristics of the size distribution can be represented by the variation in a parameter, called microstructural entropy.

(3) The nearest neighbor face correlations obtained by the simulated grain structures with isotropic and anisotropic grain boundary energies are quite similar to the Aboav-Weaire relation.

\section{REFERENCES}

1) H. V. Atkinson: Acta Metall. 36 (1988) 469-401.

2) D. Weaire and J. A. Glazier: Mater. Sci. Forum 94-96 (1992) 27-38.

3) M. Hillert: Acta Metall. 13 (1965) 227-238.

4) J. E. Burke and D. Turnbull: Prog. Metal. Phys. 3 (1952) 220-292.

5) N. P. Louat: Acta Metall. 22 (1974) 721-724.

6) D. Weaire and F. Bolton: Phys. Rev. Lett. 65 (1990) 3449-3451.

7) D. Weaire and J. P. Kermode: Philos. Mag. B 48 (1983) 245-249.

8) D. Weaire and H. Lei: Philos. Mag. Lett. 62 (1990) 427-430.

9) K. Kawasaki, T. Nagai and K. Nakashima: Philos. Mag. B 60 (1989) 399-421.

10) C. V. Thompson, H. J. Frost and F. Spaepen: Acta Metall. 35 (1987) 887-890.

11) M. P. Anderson, D. J. Srolovitz, G. S. Grest and P. S. Sahni: Acta Metall. 32 (1984) 783-791.
12) D. J. Srolovitz, M. P. Anderson, P. S. Sahni and G. S. Grest: Acta Metall. 32 (1984) 793-802.

13) D. Fan and L. Q. Chen: Acta Mater. 45 (1997) 611-632.

14) D. Fan, C. Geng and L. Q. Chen: Acta Metall. 45 (1997) 1115-1126.

15) J. W. Cahn and J. E. Hilliard: J. Chem. Phys. 28 (1958) 258-267.

16) Y. Saito: An Introduction to the Kinetics of Diffusion Controlled Microstructual Evolution in Materials, (Corona Publishing, Tokyo 2000).

17) A. Kazaryan, Y. Wang, D. A. Dregia and B. R. Patten: Acta Mater. 50 (2002) 2491-2502.

18) G. B. McFadden, A. A. Wheeler, R. J. Braun, S. R. Coriell and R. F. Sekerka: Phys. Rev. E 48 (1993) 2016-2024.

19) N. Ma, A. Kazaryan, D. A. Dregia and Y. Wang: Acta Mater. 50 (2002) 2491-2502.

20) W. T. Read and W. Shockley: Phys. Rev. 78 (1950) 275-289.

21) A. A. B. Sugden and H. K. D. H. Bhadeshia: Recent Trends in Welding Science and Technology, ed. by S. A. David and J. M. Vitek, (ASM International, Ohio, USA), (1989) 273-278.

22) D. A. Aboav: Metallography 5 (1972) 251-263.

23) D. Weaire and N. Rivier: Contemp. Phys. 25 (1984) 59-99.

24) S. E. Edwards and K. D. Pithia: Physica A 205 (1994) 577-584.

25) E. A. Holm, J. A. Glazier, D. J. Srolovitz and G. S. Grest: Phys. Rev. 43 (1991) 2662-2668.

26) Y. Saito and M. Enomoto: ISIJ International 32 (1992) 267-274.

27) Y. Saito: ISIJ International 38 (1998) 559-566. 